\title{
5 The Tales of a Virus: Corona Temporalities
}

[A]s everyone with lungs breathes the space between the hands and the space around the hands and the space of the room and the space of the building that surrounds the room and the space of the neighborhoods nearby and the space of the cities and the space of the regions and the space of the nations and the space of the continents and islands and the space of the oceans and the space of the troposphere and the space of the stratosphere and the space of the mesosphere in and out.

In this everything turning and small being breathed in and out by everyone with lungs during all the moments. ${ }^{155}$

One of northern Europe's largest aquariums, which goes by the name of the Blue Planet and is situated close to Copenhagen Airport, has a loggerhead sea turtle called Gaia. When the airplanes stopped taking off and landing in spring 2020, Gaia kept circling her tank. She was born in a French aquarium and is of the species Caretta caretta, the world's largest hard-shelled sea turtle. This species is often referred to as "cosmopolitan" because it can be found across the globe - in the Atlantic, Pacific, and Indian oceans. In a way it is appropriate that this particular turtle, bred in captivity, has taken up residence close to the airport and may even feel the vibrations of the biggest planes as tiny ripples in her tank. Gaia already had a significant weight problem when she arrived. In fact, her unnamed sister died during transportation and showed massive obesity at autopsy. Now the aquarium staff are struggling to keep Gaia on a diet, and they encourage her to exercise by paddling around the tank, which you as a visitor can watch through a large glass-paneled underwater tunnel. During the first lockdown, the aquarium, which is owned by a private foundation, encouraged the public to donate money to help keep the animals alive. Reportedly, children emptied their piggy banks to save Gaia, who keeps on gaining weight. $(\mathrm{KV})$

Sewage samples have revealed that COVID-19 was spreading even before the disease had a name, already circulating and mutating. Then the infectious agents were named, brought into visibility through tests and microscopes, and analyzed and interpreted so that humans could understand their structure and effects, and possible forms of medical treatment. Hypotheses about how to end COVID-19's reign began to circulate. As the life of the virus began as a gigantic, distributed hyperobject with amorphous boundaries, a narrative also commenced that focused on identifying its origin in order to be able to mark a beginning (with a host of ensuing conspiracy theories), a middle (long and winding, unfolding exponentially, and bifurcating in various regions of the world), and an end (the form of which at the time of writing is still located in a realm of anticipation and which may continue for a long time). Moreover, this narrative depends on whether we view the story of the virus as an individualized tale that 


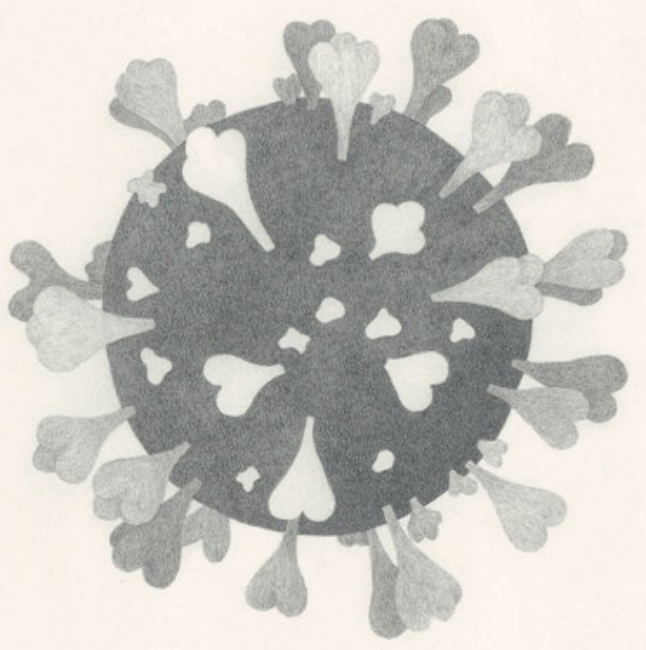

Figure 25: A drawing of the COVID-19 virus by Swedish artist Maria Finn, commissioned for this book in 2021. The image portrays the feared spikes of the virus as small, heart-shaped arms ready to touch and be touched, thus emphasizing the ambivalence of touch in the time of corona. 
may have life or death as its outcome, or as a collective narrative that is more grayscale and has many more bifurcating endings - and potential new beginnings.

The temporality of the virus is different than that of a linear narrative. Its path is outside of intentional directionalities. Despite how it felt to many of us, the virus did not arrive suddenly: it emerged - for a long time invisibly, evolving as a gigantic reproductive system of code repetitions. It spread and mutated, seeping through and between human and animal populations. In her early response to the pandemic, N. Katherine Hayles called the coronavirus a "posthuman virus," asserting that it reminds us of our interdependency with each other and the ecology of the earth, and of the urgent need to reconsider "the concepts and vocabularies with which to describe and analyze these complex interdependencies, as well as the ways in which humans, as a species, are interdependent with one another as well. The pandemic offers an opportunity to rethink the ways in which we can identify with each other and with life forms radically different from us." ${ }^{156}$ While humans have evolved by increasing their cognitive complexity as a species, "developing language with associated changes in brain and body, evolving elaborate social structures, and in very recent human history, augmenting their capacities with advanced technical devices, including artificial intelligence," ${ }^{157}$ viruses evolve toward increased simplicity, which favors the ability to replicate quickly.

The human response to the virus has been to find ways to limit contamination, complemented by techno-optimistic hopes about contact tracing apps and the race to achieve herd immunity through a vaccine. Yet as an underlying potentiality, the virus is concretely there as a gigantic distributed entity that implies a number of obstructions to a linear temporality moving forward. As Juliana Spahr notes in a poem written in the aftermath of 9/11, from which we quote in the epigraph above: "In this everything turning and small being breathed in and out by everyone with lungs during all the moments." Spahr describes a similar collapsing of time into a broad present that is also accompanied by a focus on gaps - the spaces in-between humans, and between humans and other species, which we have now learned to regard as spaces of potential contamination. In the pandemic scenario, "everyone with lungs" is at particular risk, and time is characterized by delay.

Before the coronavirus emerged, many people's foremost association with the fear of a virus would be a computer virus, or else a "viral" news story spreading beyond control and potentially reaching places far from the location of its original posting, temporally repeating itself over and over again, sometimes for years. A computer virus is a program that replicates itself, modifying 
other computer programs without the user's consent by injecting them with its own code. Its biological sibling replicates within living cells, and coronavirus has brought the biological meaning back from the virology laboratories and into public consciousness.

Notably, as we have seen throughout this book, in so many ways, the pandemic forces us to deal with a different temporality that is not a steady stream but jumps and cuts and folds itself back on top of itself. The pandemic constantly shuffles and reshuffles our sense of time, pushing the temporal experiences that have been ingrained in our everyday lives to the extreme - making more visible what we take for granted. How, then, to tell the story of a virus? What plotlines are available for this tale? These are questions with which we have grappled in this book, spurring us to go beyond our strictly academic comfort zone and embrace the associative modes of the spiraling essay as a shared form of thinking, and of writing as a fuzzy form of memoir that responds to the shared nonlinear experience of the pandemic.

However, it also sparks the question how to read a virus that spreads through mutations in genetic code that make it resemble a dynamic archive rather than a story with a satisfying end in sight. In what temporal modes do we comprehend the experience of the virus? To what temporalities does it give rise? Which narrative frameworks do we use to comprehend its existence among us - let alone represent it?

In Reading for the Plot, literary scholar Peter Brooks famously describes that which maintains the reader's interest and engagement as "narrative desire." 158 He builds on both Freud's Beyond the Pleasure Principle and Benjamin's claim in "The Storyteller" that "death is the sanction of everything that the storyteller can tell. He has borrowed his authority from death." 159 Brooks fuses these two influences to conceptualize an "anticipation of retrospection" that takes the form of a drive to ascribe meaning. ${ }^{160}$ Narrative desire is coupled with the desire for an ending as a stand-in for our own death, which we are exempt from experiencing ourselves; the desire for an ending thus comes to embody a desire to be able to ascribe meaning. In this process, the postponement of reaching the end - or the fear of reaching the end too soon - is central: "The model proposes that we live in order to die, hence that the intentionality of plot lies in its orientation toward the end even while the end must be achieved only through detour." ${ }^{161}$ Detour and delay - for instance, through repetitions or plot excursions - are central to a satisfactory plot. They are closely related to the concept of latency, 
which we have argued characterizes the pandemic experience. Living through the pandemic, we do not know how many detours still lie before us, and we are repeatedly left to wonder what tolls the pandemic is still to take on us - as individuals and as a collective. Only time will tell when and how the current emphasis on physical distancing will subside and by which forms of touch it will be replaced - some doctors, for example, hope that the practice of shaking hands will never return, as it is a way of transmitting many diseases, not just COVID-19. ${ }^{162}$

This longing for an end that can ascribe meaning seemed to be innate in many of the first responses to the pandemic. As the philosopher Emmanuel Alloa aptly remarked, the pandemic was interpreted "as a sign, profane or divine, of all the recent errors of humanity, from hypermobility to overconsumption"163 - for example, by Pope Francis. ${ }^{164}$ Reading the pandemic within a framework of guilt and Mother Nature's revenge on humanity can produce tales of Armageddon as well as hopes for what will come after. The multiple stories of dwindling pollution during lockdown, and of wild animals regaining lost territories, are examples. At other times, the news gave peculiar echoes of disaster movies about the extinction of the human race. A common pastime during the first lockdown was for people to use social media to share examples of popular culture that seemed to anticipate the events we were living through. By turning to well-known narratives in this way, we could ease the uncertainty and reinscribe what gripped us into familiar, appeasing frameworks, even if they involved gory tales. For instance, Andrea Bocelli's "Music for Hope” concert from the Duomo in Milan on Easter Sunday 2020 became the largest livestream event on YouTube to that date. A tiny figure on the empty square, the blind tenor stood before the grand cathedral whose architecture highlighted the frailty of human existence, and he sang "Amazing Grace," whose lyrics propose a temporality that stretches beyond the individual life, indeed beyond the existence of the earth. The pathos-laden live performance was interspersed with footage from cities around a world empty of people, accentuating the sensation that we were living through the last days on earth, which the Easter narrative only served to emphasize.

Yet, at the same time as the narrative of Armageddon looms large, the future is already folded back onto the present. Contact tracing tries to contain the spread of the virus by tracking past interactions in order to prevent future outbreaks, highlighting how the latent presence of the virus during the incubation period determines futures yet unknown. Moreover, archives of the future open up when genetic material is deposited for unknown later use. In a centralized country such as Denmark, the human material collected by swab is 
automatically sent to and registered in a national biobank, along with possible metadata on age, gender, address, socioeconomic situation, and more. In this situation, COVID-19 testing is an opportunity, one might say, to establish a physical imprint or genetic archive of the concrete biological material in which the virus mixes with human cells as it spreads through the population. It creates an archive of the population that will be useful for the prediction of other diseases and conditions, but also ripe for other kinds of data analysis.

Despite attempts to place the pandemic experience within well-known narratives of the end of times, the experience of living through a pandemic also reveals that many people today are not only reading for the plot and orienting themselves toward a temporality beyond earthly existence. They are also orienting themselves toward other ways of structuring the information that fills the cultural landscape of our everyday lives, going beyond a story with a beginning, a middle, and an end, and offering alternative models to accommodate the serial character of the pandemic, which relentlessly adds new and surprising chapters. One dominant information structure in contemporary culture is the social media newsfeed, which is characterized by a particular temporality that evades any sense of an ending and always has something new to offer. Media scholar Taina Bucher has named the temporal regime produced by the increasingly algorithmic media landscape "right-time." This apt notion describes the ambition of most social and news media platforms to display the right information at the right time. ${ }^{165}$

Algorithmically sorted and organized, these platforms show us what they expect us to want to read, when they anticipate that we want to read it. We may add that this reading temporality seems to revive literary theorist Wolfgang Iser's notion of the "implied reader," which describes the reader anticipated by a given text. The implied reader is a textual structure, not a real reader of flesh and blood conditioned by history and culture: "The concept of the implied reader designates a network of response-inviting structures, which impel the reader to grasp the text." ${ }^{166}$ In its algorithmic iteration, the implied reader has become a data double constructed on the basis of the reader's past actions, making it simultaneously individual and collective. Although its algorithmic iteration is also not identical with a particular historically and culturally conditioned reader, this algorithmically anticipated reader is predicated on the input that a particular reader provides to the algorithm. Moreover, the network of "response-inviting structures" not only impels us to grasp the text, but also 
anticipates when we will grasp it, thus folding different temporalities on top of each other: the timestamp when the text was posted, but also the time when it emerges in my newsfeed, which may be instantly, or hours or days later - or not at all. The incubation period of a post's emergence is dependent on the shape and form of my algorithmically calculated data double.

A key constituent of COVID-19, like many other infections, is that the illness is often characterized by a delay - not only between the moment of infection and the emergence of the first symptoms, but between hospitalization and the longer-term health effects, with many reported late sequelae. When it comes to both political and medical responses to COVID-19, the experience of time as delayed repeatedly resurfaces as a condition and challenge to overcome. We see it in the calculations of the length of time lapse between two doses of vaccine, and in the possibility of extending this period in order to be able to give the vaccine to more people - which in the winter of 2020-2021 ran counter to the spread of new, vastly more infectious, and possibly vaccine-resistant variants. ${ }^{167}$ The possible eruption of such new variants, which can quickly lead to an exponential growth in infections and make epidemiological management difficult, emphasizes latency, here as an invisible contest between different COVID-19 variants. ${ }^{168}$

We may thus argue that the temporality that foregrounds the latent and the delayed is a more apt structure through which to grasp the pandemic situation than is a story with a beginning, a middle, and an end. Yet linearity is hard to shake, perhaps especially in times of crisis, which designates a different temporal state than the catastrophic. ${ }^{169}$ Linearity gives us something to hold onto a sense that the world is moving forward when it otherwise seems to have come to a halt. When the news kept rolling in as the pandemic gained ground around the world, something interesting happened on most online news sites: the algorithmically structured and individually tailored "front pages" were replaced with a newsfeed structure, with one story under another. However, these were ordered in a linear manner that depended on when the news ticked in, and not on a sense of individualized "right-time." While the front-page structure is modeled on its print media predecessors, the newsfeed that materialized was more modeled on social media newsfeeds that could be scrolled, displaying seemingly endless corona-related news, but without the individualized tailoring, as if to say that news of the pandemic was rightly timed for everyone. The reintroduction of the posts' linear temporality evoked a keen awareness of time zones: we woke up to an avalanche of news from the zones where people had been awake while we slept. This only emphasized the experience of a truly global pandemic, and ironically of the conflation of time into a broad present 
where the sun never sets and there is no need to close your book and turn off the light.

Admittedly, the experience of time during lockdown varied markedly depending on whether you worked in a hospital, had children or elderly family members in your household, or had a job that could be performed digitally. Not everyone experienced more time on their hands, but many people definitely had to deal with time in a different way. Literary scholars Irina Dumitrescu and Caleb Smith have compared the experience of lockdown to the lives of preindustrial monks in the fourth and fifth centuries. They recount John Cassian's writings about the difficulty of sustaining their solitude and the monotony of daily life as centering on acedia, which in the monastic tradition means "lack of care." Acedia was "a roving, lonely, agitated sensation, a feeling of being unhappy in one's place that could spiral into downright depression." ${ }^{170}$ It was linked to the experience that one's ties to other people and God had been severed: "a lonely wandering of the mind." ${ }^{111}$ Manual labor or meditation helped monks to tame the demon of acedia; Dumitrescu and Smith argue that the gardening, sewing, or baking that many people took up during lockdown served a similar function, appeasing demons, keeping hands busy, and bestowing a sense of purposefulness. Their hope is that this alternative experience of time may reveal something other than the degree to which we have internalized capitalist logics and feel compelled to produce even in a lacuna. Perhaps it can teach us how to deal with the experience of disorder, buzzing news updates, and time "flowing out of form" that collapses together work, rest, and play, an experience that became acute during the pandemic but had already been part of contemporary life for many people. May we hope for a temporal regime that is less set on moving forward and more set on staying with the trouble of the everyday? One that seeks to provide a space for care, rather than the lack of care that characterizes acedia?

In the 1960s, the French writer Georges Perec proposed the terms infraordinary and endotic to describe that which is neither extraordinary nor exotic but still deserves our heightened attention. Perec laments: "What speaks to us is always the big event, the un-toward, the extra-ordinary: the front-page splash, the banner headlines." ${ }^{172}$ He calls instead for an alert, attentive mode that rekindles the astonishment we feel when we encounter something for the first time: "What we need to question is bricks, concrete, glass, our table manners, our utensils, our tools, the way we spend our time, our rhythms. To question 
that which seems to have ceased forever to astonish us. We live, true, we breathe, true; we walk, we open doors, we go down staircases, we sit at a table in order to eat, we lie down on a bed in order to sleep. How? Why? Where? When? Why?" ${ }^{173}$ Perec calls for a mode of experience that is as attentive to the "small" and habitual as it is to the extraordinary and gigantic.

In giving the mundane as much attention as the everyday, he is doing something very different than the feminist readings of infrastructure that call attention to things that escape public awareness and seep into the background - things that are not deemed noteworthy until they fail or collapse. ${ }^{174}$ Sociologist Susan Leigh Star talks about infrastructure as "an embedded strangeness, a second-order one, that of the forgotten, the background, the frozen in place."175 American novelist Ursula K. Le Guin also points to this when she writes that the history of mankind is written from the perspective of the man as hunter, and that it tracks history as a series of advancements such as the control of fire and the invention of the spear and other weapons. She reminds us that these inventions in and of themselves would have been insignificant if there would have been no "carrier bag" or container to carry back the prey, and in this way she points to the significance of the work of the gatherer. ${ }^{176}$

But rather than insisting that we bestow the same amount of attention on the uneventful as we do on the extraordinary, these approaches address the mundane in its own habitat, and this is an important difference in terms of how to tackle that which surrounds us and has become habitual. It is about asking, as we did in Chapter 3, "why do you do it like this?" The social media newsfeed performs a similar drumming-up of expectations regarding the mundane as Perec does with his microsociology: it features everyday, mundane experiences through the vocabulary of the extraordinary. It does not let the everyday remain on the periphery of our attention, but promotes it to the status of infraordinariness, post after post after post. It makes for a reading mode that encourages a distracted skimming enacted by the repetitive brushing of the finger against the screen, pushing what has been consumed upward in a slow, caressing, anxious, bored, or impatient manner, depending on the mood of the reader and how interesting the news on display is. ${ }^{177}$ The ordinary comes to bear a significance that is otherwise bestowed only on extraordinary events; the small takes on gigantic proportions. However, if we regard social media newsfeeds overall as amalgamations of the mundane that cancel each other out in their competition for our attention, we may read them through a feminist lens, which treats the mundane as an object of interest without having to remove it from its natural habitat and elevate it to a status of the extraordinary. 


\section{DAYS INTO \\ CORONA SHUTDOWN: \\ 47}

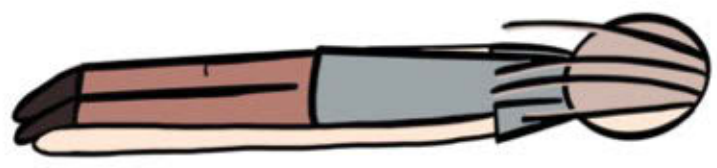

When will theis and?

KRedReggae

Figure 26: During the first lockdown in Denmark, Danish artist Rikke Reimann posted a new drawing on her Instagram profile every day, counting the days. Image Credit: Rikke Reimann 
In curious ways, these modes of reading and absorbing the news reflect and accentuate the temporal experience that lockdowns bring about: the heightening of attention to the mundane and the everyday because we are locked in, with fewer points of social interaction, makes everything that happens take on new significance, but simultaneously cancels out the sense that anything is "an event" that sets it apart from what happened yesterday or the day before. In spring 2020, many local archives across Denmark encouraged people to send in their diaries, social media posts, photos, and scribblings to chronicle the event of lockdown. On one hand, this archival practice marked the lockdown and the virus as something out of the ordinary - something that in archival terms was deemed important enough to solicit documentation. At the same time, it made for an amalgamation of everyday events that turned the focus toward the infrastructure of the everyday, at a time when that everyday was anything but ordinary.

What the archives were picking up on and trying to harvest was the marked rise in people who were chronicling their lockdown experiences on social media, counting the days. For instance, writer and artist Rikke Reimann's Instagram profile, red.reggae, counted the days by posting drawings every day for the first period of lockdown. Adding such linearity to the passing of the days, which might otherwise risk collapsing into each other, helped to structure time, and created pockets of commonality where people could partake in a communal counting of days. It also made for weird loops, because the lockdown counts started at different times in different parts of the world, even though people were using the same hashtags. Not unlike news sites' introduction of linearity to structure the influx of virus-related news, the lockdown and the impetus to chronicle it somehow formed a resistance to the "right-time" logic of social media: the counting of days disrupted the flow of algorithmically tailored news, making that temporal practice visible by inserting a logic of linearity and progression into it. There is a curious coupling between social media as sites for articulating the mundane and different experiences of time on one hand, and on the other hand the experience of lockdown as entailing different needs in terms of temporality (counting toward an end, or letting it all collapse together). 


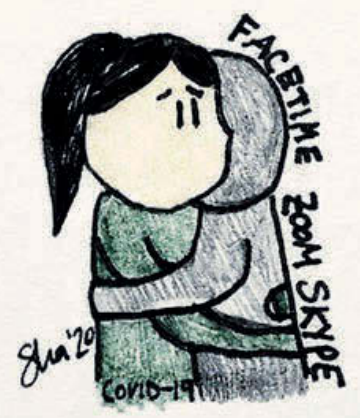

Figure 27: In March 2020, the Indiana Historical Society launched a collecting initiative to document the COVID-19 pandemic and its impact. The donor's description of this image reads:

"I decided to stay in Pittsburgh in my little studio apartment to protect my parents, both of whom are in Indiana. My phone and the internet have always been a prominent form of communication for me, but I have never preferred it to be my primary method of socializing. Human touch and in-person connection are so important, and the decision to not come back to Indiana, to my family, has been really hard. I miss hugs. A lot. But I know I'm being responsible. Having the technology we have now to still be able to see my loved ones' smiles and laugh virtually with them have become a new form of hug for me. While I don't wish this to be the norm long term, it'll do for now."

Image Credit: “COVID Hug” by Sarah Sha, Indiana Historical Society 
In an interview in March 2021, the Norwegian author Linn Ullmann explained why she had titled the podcast she started when the pandemic hit "How to Proceed": "Both in writing and in reading, we talk a lot about beginnings, and we talk a lot about how something ends, but I often think the question is 'how to proceed,' or 'what now,' or 'what do I do today,' or 'how do I do this,' or 'how do I go on now.'" 178 Delay and the long and winding middle are the temporality in which we are caught. This is the temporality of the everyday, of getting up in the morning and proceeding. The virus seems to create a particular temporality that this book has argued is characterized by latency as the overall trope. Yet the sensation of pandemic stuckness that we have identified also makes for the collision of different temporal regimes and narrative formulas that rub against each other when we try to make sense of these experiences, linearity and the desire for an end being perhaps the most persistent. Probing the available plotlines of the experience of the first year of the COVID-19 pandemic, and the modes of information structuring that inform our engagement with a crisis such as this, as we have done in this chapter, allows us to see the ways in which the pandemic simultaneously taps into and enhances existing temporal modes that structure our lives, while also forcing us to question these narratives and possibly start carving out spaces for alternative ways of engaging with the present and imagining alternative futures. Asking "how to proceed," without knowing the next step. 
